\title{
Corpus Callosum Atrophy in a Patient with Neuromyelitis Optica
}

\author{
K. Alikhani, D.H. Lee, M. Kremenchutzky
}

Can. J. Neurol. Sci. 2011; 38: 165-167

Neuromyelitis optica (NMO) is an inflammatory demyelinating disease of the central nervous system characterized by recurrent episodes of optic neuritis and transverse myelitis ${ }^{1}$. The most prominent magnetic resonance image (MRI) finding in NMO is longitudinally extensive transverse myelitis. We report a woman with corpus callosum atrophy on brain MRI, who was initially diagnosed as having multiple sclerosis (MS).

\section{CASE RePORT}

This otherwise healthy Caucasian woman had presented at the age of 20 with left optic neuritis, which had resulted in complete loss of vision in this eye, followed by several episodes of transverse myelitis and contralateral optic neuritis, with partial recovery. One year later, she was diagnosed with MS, and treated with varying doses of oral prednisone and azathioprine. She continued to have repeated episodes of optic neuritis and transverse myelitis. Five years since onset, interferon beta1-b $250 \mathrm{mcg}$ SC qod was started in combination with azathioprine. She had no further neurologic episodes over the following year, until azathioprine was discontinued due to neutropenia, and interferon beta1-b was continued at half dose. Subsequently, she had recurrent episodes of optic neuritis and transverse myelitis, treated with high dose steroids at least twice a year. Eight years after presentation, interferon beta1-b was discontinued, and she started glatiramer acetate $20 \mathrm{mg}$ qd. She continued to experience optic neuritis at least twice a year, treated with high dose steroids. When she presented to us 18 years after initial presentation, her best visual acuity was finger counting at three meters on the right, and no light perception on the left. Pupils were dilated and unreactive to light on the left, with a sluggish response to light on the right. She had left exophoria and nystagmus in all directions of gaze. She had a spastic quadriparesis, and was able to take a few steps with a walker. Laboratory investigations revealed weakly positive ANA, elevated rheumatoid factor of $158 \mathrm{IU} / \mathrm{mL}$, negative anti-dsDNA, negative anti-ENA screen, normal vitamin B12 levels. Serum NMO antibody was reported positive by Mayo Clinic laboratory in Rochester Minnesota, USA.

Brain MRI showed callosal atrophy, centrally in the body. A few nonspecific high T2 signal white matter abnormalities were seen in the deep white matter of the hemispheres. No lesions were seen in the posterior fossa or the periventricular areas. The spinal cord was markedly atrophic, and no signal change was seen in the spinal cord. No abnormal enhancement was seen with Gadolinium (Gd) injection. (Figure)

We recommended discontinuation of glatiramer actete; azathioprine was initiated. At the time of preparation of this manuscript, the patient has been neurologically stable for more than 12 months after having started azathioprine.

\section{DiscuSSION}

Neuromyelitis optica was long thought to be a variant of MS, but it is now recognized that clinical, laboratory, immunological, and pathological features distinguish NMO from $\mathrm{MS}^{1}$. Detection of NMO-IgG, a highly specific serum autoantibody directed against aquaporin-4 (AQP4) water channel, provides further evidence that $\mathrm{NMO}$ is distinct from MS and has helped define an NMO spectrum of disorders ${ }^{2}$.

The most reliable element in the diagnosis of NMO is the longitudinally extensive transverse myelitis lesion. Spinal cord lesions characteristically span three or more contiguous vertebral segments, and involve preferentially the central gray matter of the spinal cord ${ }^{1,3-5}$

At onset, brain MRI in NMO is typically normal except for Gd enhancement of the optic nerve during an acute optic neuritis. However, the absence of clinical disease outside optic nerves or the spinal cord is no longer considered necessary for the diagnosis of NMO. ${ }^{6}$ This has been reflected in the most recent revision of the diagnostic criteria for definite NMO, which require optic neuritis, myelitis, and at least two of the following supportive criteria: MRI evidence of a contiguous spinal cord lesion three or more segments in length, onset brain MRI nondiagnostic for MS, or NMO-IgG seropositivity. ${ }^{7}$

Non-specific white matter lesions ${ }^{8}$, and brainstem lesions in isolation or as extension of cervical lesions ${ }^{8,9}$ have been reported in NMO. Later in the course of NMO, lesions in the brain MRI are seen in $60 \%$ of patients ${ }^{10}$. Some studies have suggested that certain brain lesions occur specifically in NMO, including lesions in the periaqueductal, hypothalamic and periventricular regions, and bilateral, longitudinally extensive lesions in the cerebral white matter ${ }^{11-13}$.

Corpus callosum involvement is considered a sensitive and specific indicator of multiple sclerosis ${ }^{14}$, and callosal atrophy is common in $\mathrm{MS}^{15}$. The frequency and characteristics of callosal

From the Department of Clinical Neurological Sciences (MK), Department of Diagnostic Radiology (DHL), the University of Western Ontario, London, Ontario; Department of Clinical Neurosciences (KA), University of Calgary, Calgary, Alberta, Canada.

Received June 9, 2010. Final Revisions Submitted August 13, 2010. Correspondence to: Katayoun Alikhani, Room 1007B Health Sciences Centre, 3330 Hospital Drive NW, Calgary, Alberta, T2N 4N1, Canada. 

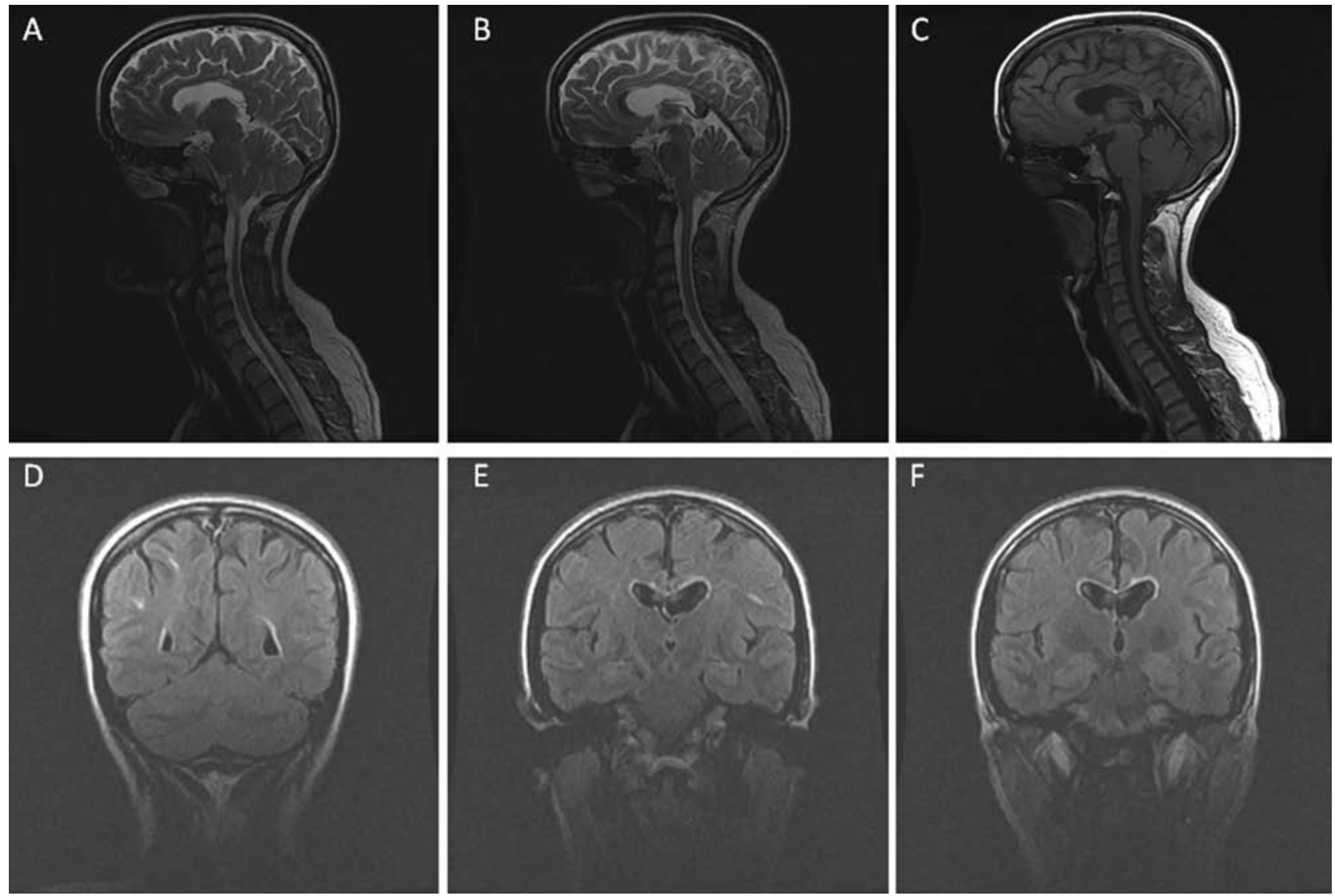

Figure: Brain and cervical MRI findings. T2 sagittal MRI demonstrating thining and hyperintensity in the corpus callosum body. Note marked thinning of spinal cord. (A, B). T1 sagittal MRI showing marked callosal atrophy $(C)$. FLAIR coronal images showing foci of T2 hyperintensity in the subcortical and periventricular white matter $(D, E)$, and abnormal signal in the left side of the corpus callosum $(F)$.

lesions in NMO have not been studied extensively. Matsushita et al have reported a higher frequency of anterior periventricular linear lesions lining the corpus callosum and lateral ventricles in patients with opticospinal MS compared to the conventional form $^{16}$. Nakamura et al reported callosal lesions in four (18.2\%) of $22 \mathrm{NMO}$ patients. All of these patients were positive for NMO- $\operatorname{IgG}^{17}$.

The mechanisms of CC involvement in NMO are unclear. It is possible that NMO autoantibodies down regulate AQP4 expression on astrocytic foot processes causing an astrocytopathy ${ }^{18}$. In rats, AQP4 is more densely distributed on the astrocytes in the CC than in the cerebral cortex ${ }^{19}$.

The extensive callosal atrophy seen in this case may have been the long term consequence of previous inflammatory lesions in the corpus callosum. Furthermore, NMO-IgG was positive eighteen years after presentation, in the absence of MRI evidence of active inflammation. To our knowledge, this is the first report of corpus callosum atrophy in this condition, which demonstrates an example of brain MRI abnormalities in NMO. It also illustrates the clinical utility of testing for NMO-IgG in the diagnosis of this severe inflammatory demyelinating condition.

\section{References}

1. Wingerchuk DM, Hogancamp WF, O'Brien PC, et al. The clinical course of neuromyelitis optica (Devic's syndrome). Neurology. 1999; 53: 1107-14.

2. Lennon VA, Wingerchuk DM, Kryzer TJ, et al. A serum autoantibody marker of neuromyelitis optica: distinction from multiple sclerosis. Lancet. 2004; 364: 2106-12.

3. de Seze J, Stojkovic T, Ferriby D, et al. Devic's neuromyelitis optica: clinical, laboratory, MRI and outcome profile. J Neurol Sci. 2002; 197: 57-61.

4. Papais-Alvarenga RM, Miranda-Santos CM, Puccioni-Sohler M, et al. Optic neuromyelitis syndrome in Brazilian patients. J Neurol Neurosurg Psychiatry. 2002; 73: 429-35.

5. Nakamura M, Miyazawa I, Fujihara K, et al. Preferential spinal central gray matter involvement in neuromyelitis optica: an MRI study. J Neurol. 2008; 255: 163-70.

6. O'Riordan JI, Gallagher HL, Thompson AJ, et al. Clinical, CSF, and MRI findings in Devic's neuromyelitis optica. J Neurol Neurosurg Psychiatry. 1996; 60: 382-7.

7. Wingerchuk DM, Lennon VA, Pittock SJ, et al. Revised diagnostic criteria for neuromyelitis optica. Neurology. 2006; 66: 1485- 9.

8. Misu T, Fujihara K, Nakashima I, et al. Intractable hiccup and nausea with periaqueductal lesions in neuromyelitis optica. Neurology. 2005; 65: 1479-82.

9. Chalumeau-Lemoine L, Chretien F, Gaelle Si Larbi A, et al. Devic disease with brainstem lesions. Arch Neurol. 2006; 63: 591-3. 
10. Pittock SJ, Lennon VA, Krecke K, et al. Brain abnormalities in neuromyelitis optica. Arch Neurol. 2006; 63: 390-6.

11. Pittock SJ, Weinshenker BG, Lucchinetti CF, et al. Neuromyelitis optica brain lesions localized at sites of high aquaporin 4 expression. Arch Neurol. 2006; 63: 964-8.

12. Poppe AY, Lapierre Y, Melancon D, et al. Neuromyelitis optica with hypothalamic involvement. Mult Scler. 2005; 11: 617-21.

13. Nakashima I, Fujihara K, Miyazawa I, et al. Clinical and MRI features of Japanese MS patients with NMO-IgG. J Neurol Neurosurg Psychiatry. 2006; 77: 1073-5.

14. Gean-Marton AD, Vezina LG, Marton KI, et al. Abnormal corpus callosum: a sensitive and specific indicator of multiple sclerosis. Radiology. 1991; 180: 215-21.

15. Dietemann JL, Beigelman C, Rumbach L, et al. Multiple sclerosis and corpus allosum atrophy: relationship of MRI findings to clinical data. Neuroradiology. 1988; 30: 478-80.
16. Matsushita T, Matsuoka T, Ishizu T, et al. Anterior periventricular linear lesions in optic-spinal multiple sclerosis: a combined neuroimaging and neuropathological study. Mult Scler. 2008; 14: 343-53.

17. Nakamura M, Misu T, Fujihara K, et al. Occurrence of acute large and edematous callosal lesions in neuromyelitis optica. Mult Scler. 2009; 15(6): 695-700.

18. Lennon VA, Kryzer TJ, Pittock SJ, et al. IgG marker of optic-spinal multiple sclerosis binds to the aquaporin-4 water channel. J Exp Med. 2005; 202: 473-7.

19. Badaut J, Verbavatz JM, Freund-Mercier MJ, et al. Presence of aquaporin-4 and muscarinic receptors in astrocytes and ependymal cells in rat brain: a clue to a common function? Neurosci Lett. 2000; 292: 75-8. 